\title{
On the time resolution of event-related desynchronization: a simulation study
}

\author{
Thomas R. Knösche ${ }^{a, *}$, Marcel C.M. Bastiaansen ${ }^{b}$ \\ ${ }^{a}$ MEG Group, Max Planck Institute of Cognitive Neuroscience, PO Box 500355, 04303, Leipzig, Germany \\ ${ }^{\mathrm{b}}$ Max Planck Institute of Psycholinguistics, PO Box 310, 6500 AH Nijmegen, The Netherlands
}

Accepted 26 February 2002

\begin{abstract}
Objectives: To investigate the time resolution of different methods for the computation of event-related desynchronization/synchronization (ERD/ERS), including one based on Hilbert transform.

Methods: In order to better understand the time resolution of ERD/ERS, which is a function of factors such as the exact computation method, the frequency under study, the number of trials, and the sampling frequency, we simulated sudden changes in oscillation amplitude as well as very short and closely spaced events.

Results: Hilbert-based ERD yields very similar results to ERD integrated over predefined time intervals (block ERD), if the block length is half the period length of the studied frequency. ERD predicts the onset of a change in oscillation amplitude with an error margin of only 10 $30 \mathrm{~ms}$. On the other hand, the time the ERD response needs to climb to its full height after a sudden change in oscillation amplitude is quite long, i.e. between 200 and $500 \mathrm{~ms}$. With respect to sensitivity to short oscillatory events, the ratio between sampling frequency and electroencephalographic frequency band plays a major role.

Conclusions: (1) The optimal time interval for the computation of block ERD is half a period of the frequency under investigation. (2) Due to the slow impulse response, amplitude effects in the ERD may in reality be caused by duration differences. (3) Although ERD based on the Hilbert transform does not yield any significant advantages over classical ERD in terms of time resolution, it has some important practical advantages. (C) 2002 Elsevier Science Ireland Ltd. All rights reserved.
\end{abstract}

Keywords: Event-related desynchronization; Brain oscillations; Non-phaselocked activity; Hilbert transform; Electroencephalographic rhythms

\section{Introduction}

The analysis of stimulus or response bound changes of electroencephalographic/magnetoencephalographic (EEG/ MEG) oscillations by means of a technique known as event-related desynchronization/synchronization (ERD/ ERS, Pfurtscheller and Aranibar, 1977) can yield information about event-related brain activity that is not contained in event-related potentials (ERPs) (Pfurtscheller and Lopes da Silva, 1999a). The interest in ERD analyses has therefore been growing considerably over the past decade (cf. Pfurtscheller and Lopes da Silva, 1999b for an extensive review of this field of research). This has led to new insights about a wide range of cognitive functions (e.g. Pfurtscheller and Berghold, 1989; Pfurtscheller and Neuper, 1994; Neubauer et al., 1995; Bastiaansen et al., 1999; Klimesch, 1999; Leocani et al., 1999).

\footnotetext{
* Corresponding author. Tel.: +49-3425-8875-33; fax: +49-3425-887511.

E-mail address: knoesche@cns.mpg.de (T.R. Knösche).
}

A disadvantage of the ERD technique, however, is that it has a relatively poor temporal resolution. In order to obtain statistically reliable responses, one has to integrate over relatively long time intervals ${ }^{1}$. The poor temporal resolution seriously limits the applicability of ERD analyses, in that (1) it hampers the study of ERD onset/offset latencies, which may prove to be valuable, for example, in studying movement disorders (cf. Defebvre et al., 1998; Magnani et al., 1998) and (2) it makes ERD less suitable for studying cognitive processes such as linguistic or attentive processes, since these can often be expected to occur in a millisecond time frame. It would therefore certainly be desirable to optimize the temporal resolution of the ERD.

\footnotetext{
1 Typically, in ERD computation intervals with lengths of 125 or $250 \mathrm{~ms}$ have been used in the literature (Pfurtscheller, 1999). Somewhat surprisingly, such intervals are used regardless of whether low frequency (e.g. 4 $7 \mathrm{~Hz}$, cf. Klimesch et al., 2001) or higher frequency (e.g. $15-26 \mathrm{~Hz}$, Pfurtscheller et al., 1996) EEG dynamics were under study, therewith overlooking the fact that time resolution may be improved when higher frequencies are studied.
} 
It has been proposed previously by Clochon et al. (1996) that using a signal envelope, obtained by computing the Hilbert transform, leads to a significant improvement of the temporal resolution of the ERD. Indeed, they demonstrated both analytically and experimentally that the ERD computed in time intervals (blocks) of $250 \mathrm{~ms}$ is essentially a time integration of the ERD computed with the Hilbert transform (HB-ERD). This method, which has subsequently been used repeatedly in psychophysiological research (e.g. Etévenon, 1997; Yordanova and Kolev, 1998; Kolev et al., 1999; Clochon et al., 1996), claimed that the HB-ERD has a millisecond time resolution, or at least a time resolution, which is "only restricted by the sampling rate" (Clochon et al., 1996; p. 126). Other researchers using the HB-ERD assume a millisecond time resolution as well (Kolev, personal communication).

This may not be a correct assumption. Admittedly, the signal that results from the computation of the HB-ERD has the same sampling frequency as the original signal. However, the true time resolution of the HB-ERD is inherently poorer than that. At the single-trial level it is at best half a period of the highest frequency contained in the signal, since the signal envelope connects each 'peak' of the filtered and rectified sinusoidal signal. But it is not clear what time resolution is obtained when averaging the HB-ERD over a number of single trials.

The main purpose of this paper is to gain a better understanding of the time resolution of the various ERD measures. A number of questions are relevant for this purpose, which will be answered by computing the ERD on simulated data, using realistic signal-to-noise ratios (SNR). First, and perhaps most importantly, the claim of Clochon et al. (1996) that HB-ERD has a better time resolution than 'classical' ERD, i.e. ERD computed with blocks of predefined length, has to be thoroughly evaluated. This will be done by comparing the time resolution of HB-ERD with that of the classical ERD, using different frequencies and different block lengths. Second, it has to be established how the time resolution of ERD varies as a function of the frequency under study, i.e. exactly to what extent the time resolution improves with increasing frequency. Finally, it is of interest to investigate how the time resolution of both the HB-ERD and classical ERD behaves as a function of the number of trials.

In order to compare the time resolution of different methods, we need an objective quantification of this time resolution. Therefore, we chose to address 3 basic dimensions on which to evaluate an abstract concept such as time resolution. First, the accuracy of the various ERD methods will be investigated. Here, we define accuracy as the difference between the actual (in this case, simulated) onset of the desynchronization and the response of the ERD measure, in other words: how accurately can we predict the actual onset of a phenomenon (that is, a change in amplitude of an oscillation) from the ERD response. Second, the step response, i.e. the rising or falling time of the HB-ERD will be quantified. And finally the sensitivity of the HBERD will be addressed, i.e. how short-lasting and how closely spaced may modulations in amplitude of the oscillatory activity be in order to be accurately and reliably distinguished by the HB-ERD?

\section{Methods}

\subsection{ERD computation with Hilbert transform (HB-ERD)}

The computation of ERD according to the original publication of Pfurtscheller and Aranibar (1977) is based on the following steps: (1) band pass filtering of the raw data, (2) squaring of the amplitude, (3) integration over equidistant time intervals (blocks), (4) averaging over trials, and (5) calculation of percentages relative to power in baseline interval. Steps (2) and (3) are intended to create a reliable estimate of the raw signal power, which then will add up in the average over trials, regardless of the phase of the original signal. The key question that always has to be answered is the one for the length of the integration blocks. Too short blocks result in an oscillating and unstable ERD, too long ones reduce the temporal resolution unnecessarily.

Clochon et al. (1996) have proposed an alternative way to obtain a measure that can be averaged over trials regardless of phase: the amplitude envelope analysis, based on the Hilbert transform. Fig. 1 summarizes the main steps of this method.

This measure computes a curve with the same sampling rate as the original data, which connects the peaks of the squared or rectified signal. Fig. 2 depicts an example. In this study, we will investigate the temporal properties of the HBERD method by means of simulations.

\subsection{Simulations}

\subsubsection{Simulation 1 - accuracy and step response}

This set of simulations is intended to investigate the behavior of the HB-ERD and classical ERD methods when used to analyze the sudden amplitude change of an oscillatory

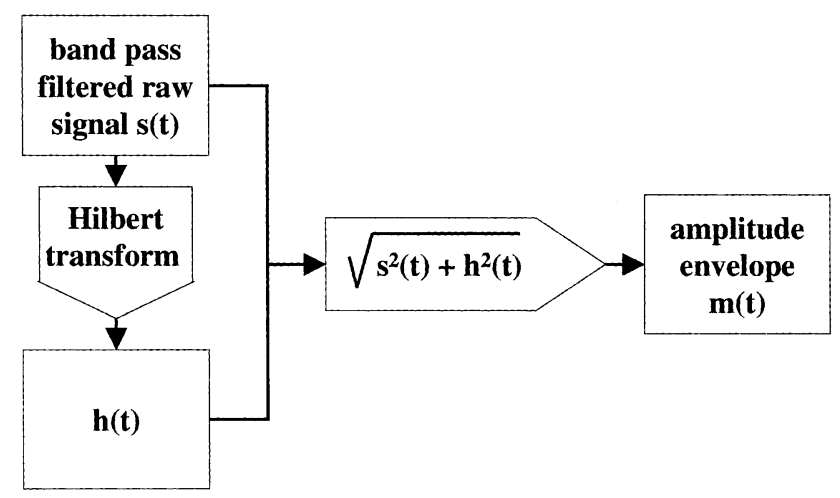

Fig. 1. Flow chart for the computation of the amplitude envelope according to Clochon et al. (1996) (simplified). 

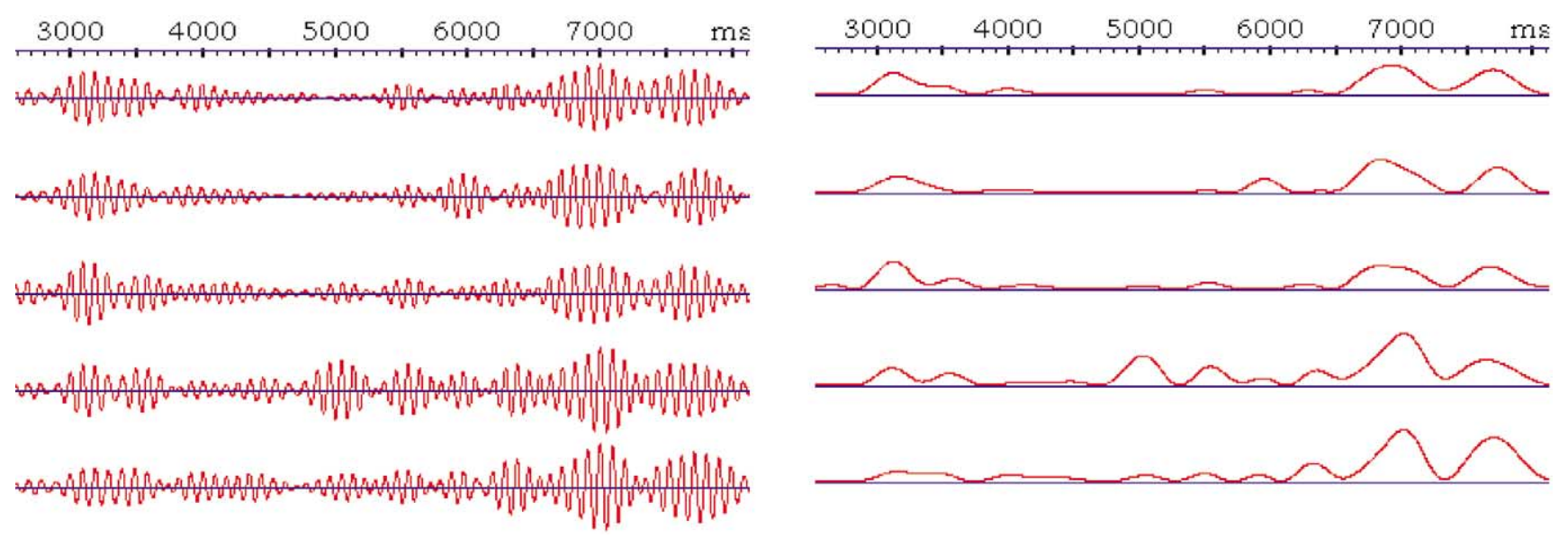

Fig. 2. Example of amplitude envelope analysis: (left) raw signal filtered to upper alpha (10-12 Hz); (right) amplitude envelope of the same signal.

signal (a 'step'). This will address the issues of accuracy and step response (i.e. the rising/falling time) of the HBERD response. We generated oscillatory test signals. Each epoch was $3 \mathrm{~s}$ long and sampled at $1 \mathrm{kHz}$. The amplitude suddenly doubled after $1 \mathrm{~s}$ and dropped back to normal after another second. A number of 1000 epochs were generated, where the phase of the oscillation varies at random between epochs. Three different variants of this test signal were made, with oscillations of $4 \mathrm{~Hz}$ (theta), $10 \mathrm{~Hz}$ (alpha), and $40 \mathrm{~Hz}$ (gamma). Both HB-ERD and classical ERD were applied to the test data sets, the latter using different integration block lengths $(1 / 20,1 / 10,1 / 5,1 / 2,1,2$, and 5 oscillation periods). Each ERD computation was carried out using the first 25, the first 50, and all 1000 epochs. After having computed all ERD results, we wanted to assess the step response with respect to (1) overall similarity of the ERD response to the true envelope of the oscillation, (2) rising/falling time, and (3) symmetry towards the true event latency. The similarity can easily be assessed by the correlation between the ERD response and the ideal oscillation envelope. However, since the various result data sets have different sampling rates (according to block length) and often also riding oscillations occur, the determination of the other two parameters is not straightforward. Hence, we sought to characterize the computed ERD step responses by an analytical function with a limited number of parameters. It turned out that the following function is a good approximation of the found responses and therefore will be used for their characterization.

$f(t)=a 1 /(1+\exp (b(c-t)))+d$

See Fig. 3 for the principal curve representing the approximating function in Eq. (1) and explanation of its parameters. The parameters were determined by a least square error fit of each of the actual step responses coming out of the simulations. The parameter $b$ gives us an indication of the rising time of the step response, which is defined as the time the ERD needs to climb from 5\% of the actual step height to $95 \%$. It can be estimated from the approximation function.

$T_{\text {trans }}=\frac{2 \operatorname{In} 19}{b}$

Parameter $c$ reveals the symmetry of the response, i.e. the relation between the actual step latency and the $50 \%$ level of the step response (the point at which the ERD reaches 50\% of its maximal value). This parameter will be used to characterize the accuracy of the ERD result.

In order to quantify the stability of the results towards noisy measurements, a subset of the simulations were repeated with Gaussian noise added to the test data sets. In order to keep the computational load at reasonable levels, we restricted this analysis to HB-ERD and classical ERD with block lengths of 0.5 and 0.05 periods. Moreover, only the use of 25 and 1000 epochs was investigated. Reliable judgement was ensured by repeating this with 10 independent noise realizations. The ratio between the variances of signal and noise was 1:2. The dependent variables Rising Time, Symmetry, and Correlation (between the true envel-

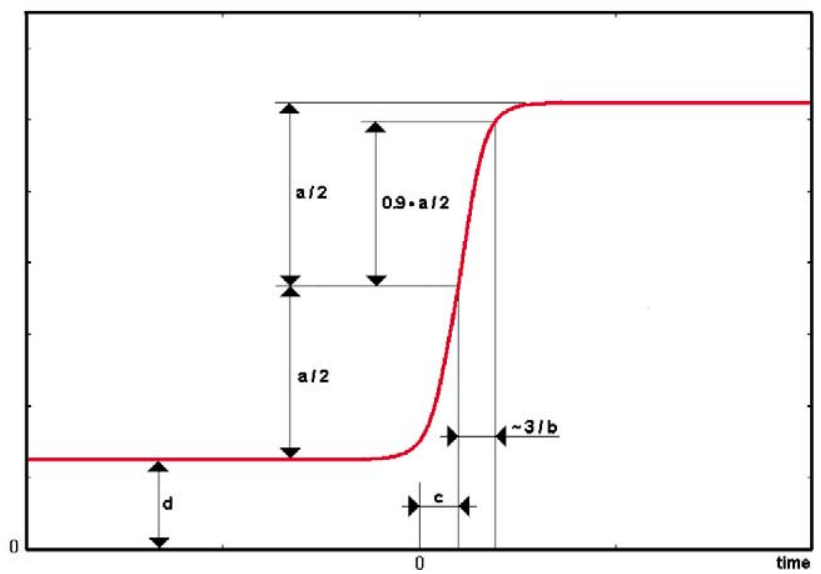

Fig. 3. Approximation function for step response: $f(t)=a 1 /(1+\exp (b(c-$ $t)))+d$. Parameters: $a$, step height; $b$, influences step width (from 0.05 to 0.95 of step height, time is $\sim 6 / b$ ); $c$, time shift (latency where half of step height is reached); $d$, initial amplitude of signal prior to transition. 


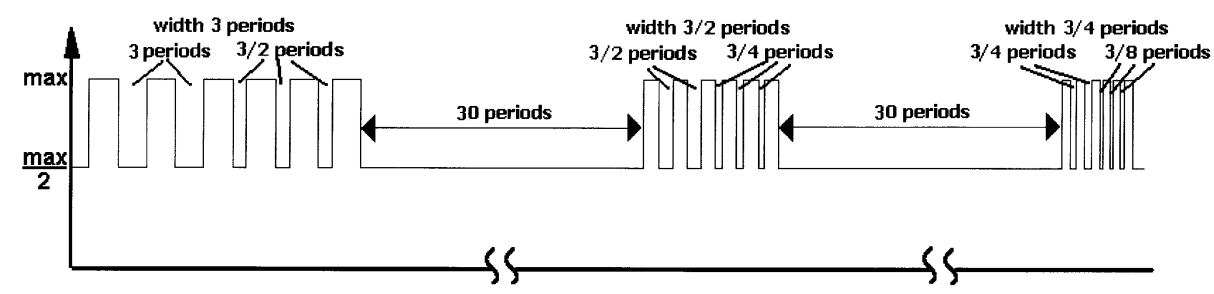

Fig. 4. Amplitudes of the test datasets for simulation 2 (sensitivity). The phase is random. The time differences are given relatively in terms of period length of the oscillations.

ope and the ERD curve) were subjected to ANOVAs for repeated measures. Within-subject factors were Method (HB-ERD, ERD with block length 0.5, ERD with block length 0.05), Frequency $(4,10,40 \mathrm{~Hz})$, and Epochs $(25$, 1000).

\subsubsection{Simulation 2 - sensitivity}

The second set of simulations is intended to investigate the HB-ERD response to brief and repetitive oscillatory events (called 'impulses' and 'impulse trains'). Here, the question of sensitivity is addressed. A test signal was created containing 3 impulse trains (see Fig. 4). It offers 6 different combinations of impulse width and inter-impulse interval length. Because of the brevity of the phenomena, also the relative sampling frequency was varied. We used relative sampling rates of 500, 250, 100, and 25 samples per period of the investigated oscillation. Note that, for example, an actual sampling rate of $1000 \mathrm{~Hz}$ means oscillation frequencies of 2, 4, 10, and $40 \mathrm{~Hz}$.

The pattern shown in Fig. 4 is repeated in 1000 epochs. Apart from the noise-free version, 20 noise realizations of each of the 4 test signals are created (10 with SNR 1 and 10 with SNR 10). The resulting input data sets were now subjected to HB-ERD, classical ERD with blocks of 0.5 periods, and classical ERD with blocks of 0.05 periods. Furthermore, each method was applied to the first 25 and to the first 250 epochs.

For analysis of the noise-free simulation results, first a visual inspection took place, judging whether or not the oscillatory impulses can be clearly identified and separated. For the noisy simulations, a $t$ test was performed in order to compare the ERD values during and between the impulses. Only if all impulses of one kind (same length and same separation) in all 10 noise realization could be distinguished significantly from both the previous and the following interimpulse interval, the ERD was considered successful in detecting the event. Note, that in this way we obtained a digital ('yes-no') answer - either an impulse is always detectable or not - in contrast to the gradual values of correlation, symmetry, and rising time used in simulation 1. Therefore, despite the factorial nature of the design, an ANOVA is not appropriate here. Since we performed a total of $5760 t$ tests $(4$ sampling rates $\times 3$ impulse widths $\times 2$ impulse intervals $\times 2$ SNRs $\times 2$ epoch numbers $\times 3$ impulses of equal sort $\times 2$ tests per impulse to either side $\times 10$ noise realizations), Bonferoni correction for multiple testing was employed. This means that for a nominal level of significance of $P<0.01$, a real level of significance of $1.74 \times 10^{-6}$ was used.

\section{Results}

\subsection{Simulation 1 - accuracy and step response}

In Fig. 5, some of the computed step responses are summarized. It can be seen that the time course is very similar for all methods, except for a ringing effect that occurs if the blocks are short with respect to the oscillation period. This effect is greatly diminished, if more epochs are taken into account. On the other hand, if the blocks are very long ( 2 or 5 periods), the final height of the step is lower (not shown in figure). HB-ERD and classical ERD with a block length of 0.5 oscillation periods show the most similar curves (see red and blue traces in Fig. 5). Fig. 6 demonstrates the high similarity of different block-based ERD variants with HB-ERD. Between classical ERD with block length of 0.5 periods and HB-ERD, there is a correlation of minimally 0.999 .

Fig. 7 additionally shows that the added noise does not change the shape of the curve of the HB-ERD. The variation between different noise realizations is relatively small, even for only 25 epochs. The same was found for block-based ERD.

The correlations between actual envelope and ERD curves, averaged over 10 noise realizations, are given in Fig. 8. The ANOVA on these correlation coefficients reveals that there is an overall trend to higher correlations with higher frequencies and (less clear) with more epochs. This is manifested by significant main effects for Frequency $(P<$ $0.0001)$ and for Epoch $(P<0.025)$. However, only for classical ERD with blocks of 0.05 periods and only for the 25 epochs case, this pattern is disturbed. In this case, higher frequencies lead to lower correlations, which is probably due to the presence of riding oscillations, as can be seen in Fig. 5. This is expressed in significant interactions Method $\times$ Frequency, Epochs $\times$ Frequency, and Method $\times$ Epochs $\times$ Frequency. (all $P$ values $<0.0001$ ).

In order to assess the relationship between the $50 \%$ level of the step response and the latency of the actual step, the parameter $c$ (representing symmetry in the approximating 


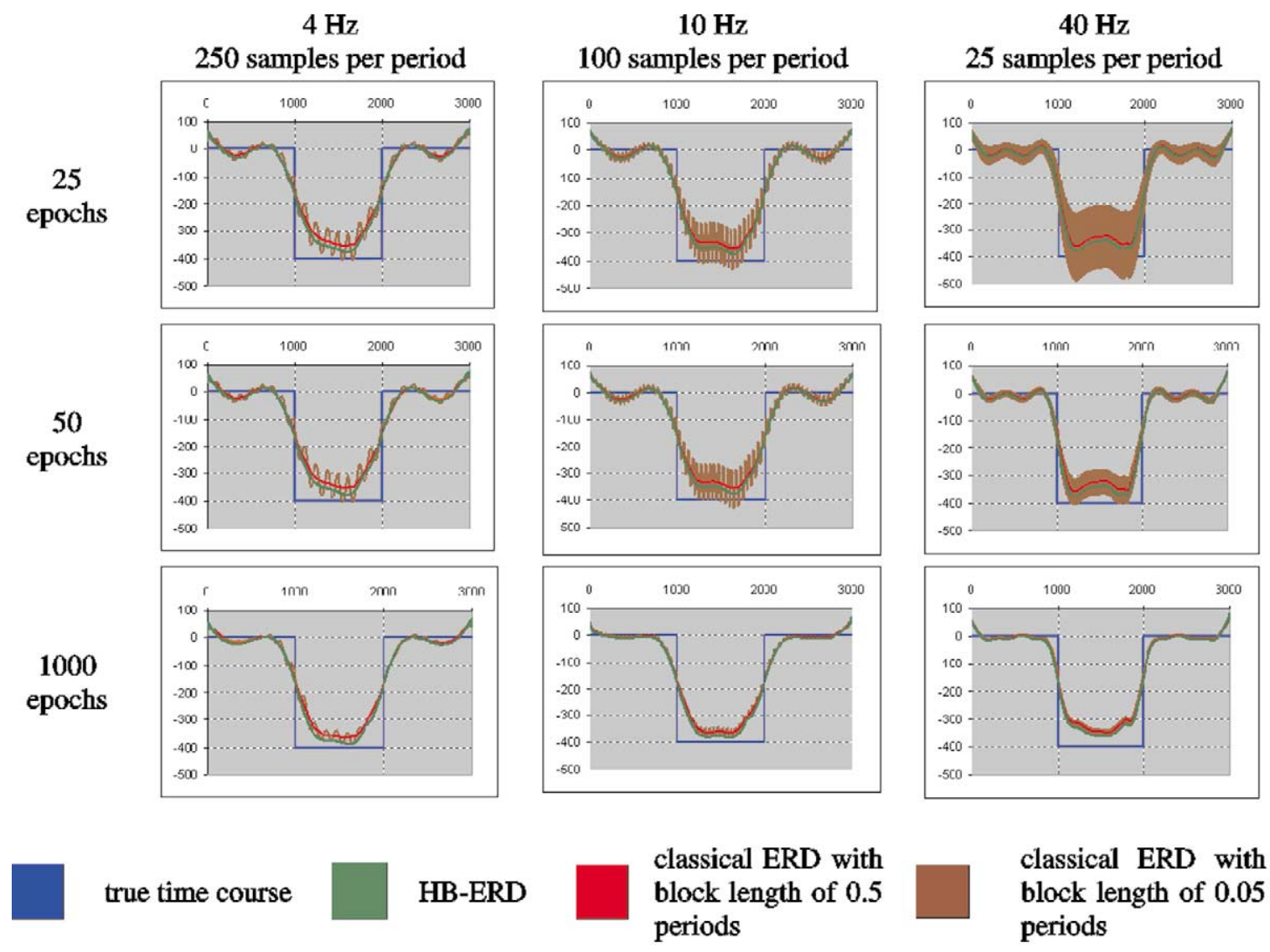

Fig. 5. Step responses of ERD for different oscillation frequencies and epoch numbers.

function, see Eq. (1)) was evaluated. Fig. 9 shows the differences between $50 \%$ level of the response and the actual step latency. It can be observed that the $50 \%$ level of the step response is generally located between 10 and $30 \mathrm{~ms}$ after the actual step latency. This means that the $50 \%$ level of the ERD response is quite a good indicator of the actual onset of the oscillatory event, at least when the onset is sudden, as in the present simulations. The difference between the various versions of ERD is quite small. The absence of main effects of the factors Method and Frequency in the ANOVA reveals that these factors do not influence the symmetry of the ERD response. The only significant effect is a main effect of

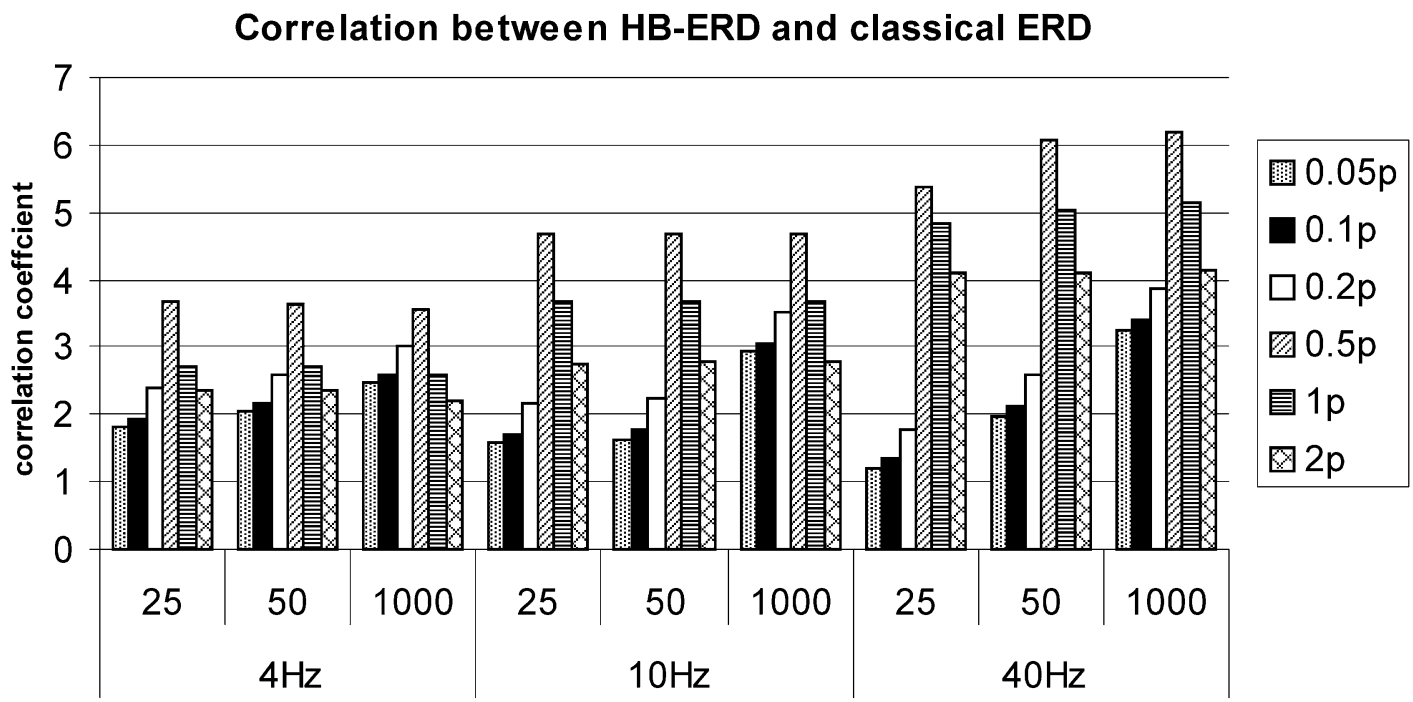

Fig. 6. Correlations between HB-ERD and classical ERD with different block length in noise-free simulations. Note the logarithmic scale. 
$4 \mathrm{~Hz}$ 250 samples per period

25 epochs

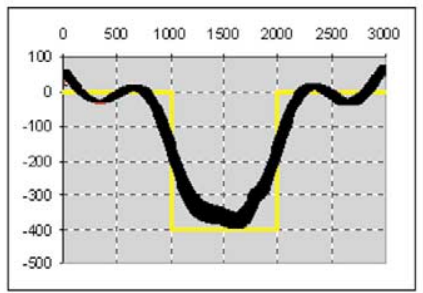

$10 \mathrm{~Hz}$ 100 samples per period

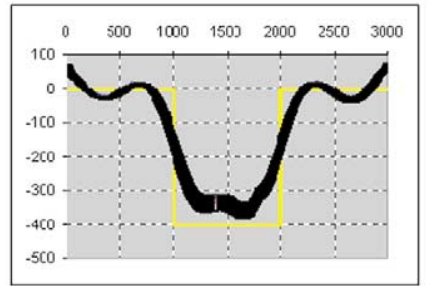

$40 \mathrm{~Hz}$ 25 samples per period

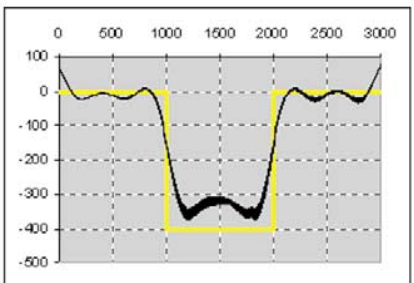

Fig. 7. Step responses of HB-ERD of noisy data (SNR 1:2, i.e. the noise variance is twice the signal variance) for different oscillation frequencies and 25 epochs (worst case). The yellow line is the true amplitude, the black band indicates the standard deviation range of the ERD.

Epoch, indicating that the ERD responses are more asymmetric (i.e. the $50 \%$ mark of the ERD response is later than the actual oscillation onset) with higher numbers of epochs $(P<0.015)$.

Finally, an interesting parameter is the transition time (see Eq. (2)), defined as the time the ERD needs to climb from $5 \%$ of the actual step height to $95 \%$.

The transition times for the various variants of ERD as well as different oscillation frequencies and number of epochs are summarized in Fig. 10. The range of transition times is between 250 and $500 \mathrm{~ms}$, which is relatively long. It is evident that there are only very small differences between the different variants of ERD. The ANOVA revealed no significant main effects or interactions concerning the factor Method. The frequency of the oscillations plays a larger role. While for $4 \mathrm{~Hz}$ (250 samples per period), the transition times are close to $500 \mathrm{~ms}$, for $40 \mathrm{~Hz}$ ( 25 samples per period) it is between 250 and $300 \mathrm{~ms}$, indicating a higher time reso- lution for higher frequencies. The ANOVA confirms this by a highly significant main effect of Frequency $(P<0.0001)$. Moreover, at higher frequencies $(10$ and $40 \mathrm{~Hz})$, the inclusion of more epochs seems to prolong the transition time, while at $4 \mathrm{~Hz}$ the number of epochs does not have any impact. This fact is reflected by a significant interaction between Frequency and Epochs $(P<0.004)$.

\subsection{Simulation 2 - sensitivity}

The results of the simulations addressing the sensitivity question for the noise-free case are depicted in Fig. 11. All 1000 epochs were used. It is evident that the quality of the results depends not only on the length of the bursts and their spacing, but also on the sampling frequency in relation to the frequency of the investigated signal, even far beyond the limits given by Shannon's theorem. In agreement with the findings in simulation 1, classical ERD with a block length

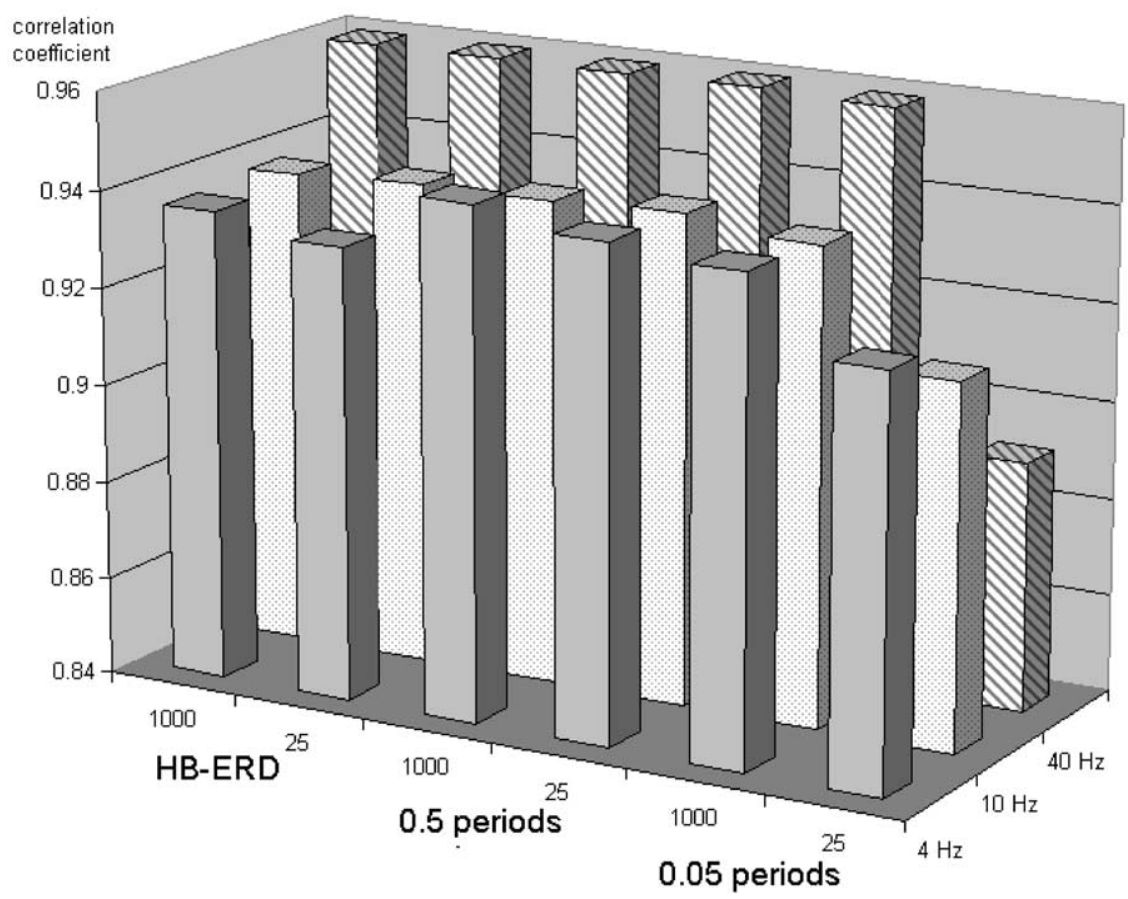

Fig. 8. Correlation between ideal box-shaped envelope of oscillation burst and ERD response. Average of 10 simulations with SNR 1:2. The horizontal axis denotes the ERD technique and the number of included epochs. 


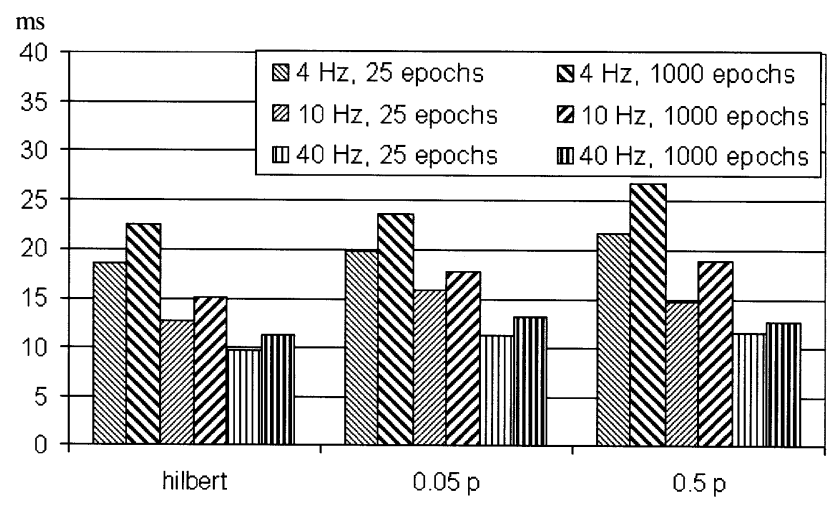

Fig. 9. Differences between 50\% level of the step response and the actual step latency in milliseconds. The letter $p$ indicates the number of periods in one block used for classical ERD.

of 0.5 periods delivers a very similar result as HB-ERD, while classical ERD with shorter blocks tends to show riding oscillations. However, if the number of sampling points per oscillatory cycle is high enough, HB-ERD is even more powerful in recovering very briefly spaced short impulses. The repetition of this simulation with only 25 epochs resulted in higher amplitude of the riding oscillation in the short block condition, but otherwise very similar curves. In Table 1, the results of the $t$ tests for the noisy case for HB-ERD are summarized. Again, we find a major influence of the length of the bursts and intervals, as well as the relative sampling rate (in relation to the oscillation frequency). The SNR and the number of trials do not seem to have a large impact on the performance of the method, at least for the values used here.

\section{Discussion}

We performed simulations in order to better understand the time resolution of ERD, and to compare classical ERD, which uses time integration over predefined intervals (blocks), with ERD based on the Hilbert transform. In a first set of simulations we addressed the questions of accuracy and step response. Accuracy was defined by how well

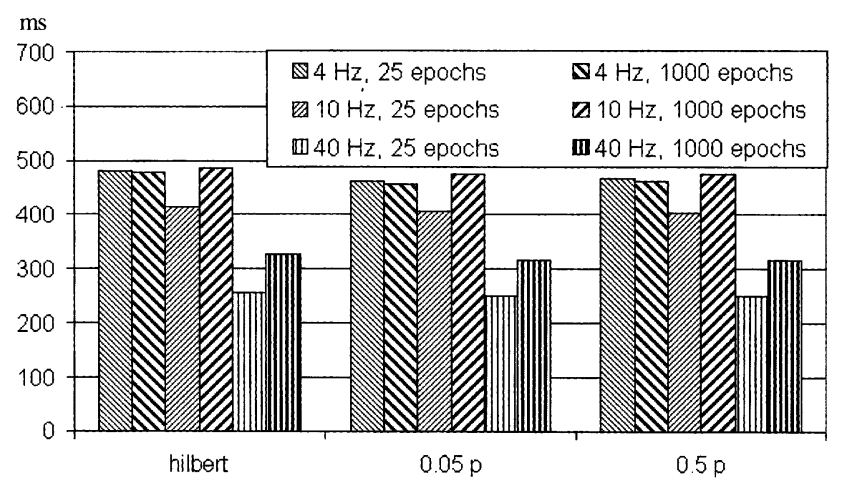

Fig. 10. Transition times as estimated from the distance between the 5 and the $95 \%$ mark of the approximation function. the ERD response could predict the onset of the oscillatory event. The step response described the time the ERD response needs to follow a sudden change in amplitude of an oscillation. Moreover, the overall similarity between the original envelope of the oscillatory signal and the ERD response was assessed using a correlation coefficient. In a second set of simulations we addressed the question of sensitivity, by subjecting trains of short oscillatory bursts to HB-ERD.

Generally speaking, the simulations showed that classical ERD/ERS with a block length of 0.5 periods of the investigated oscillations yields a response that is very similar to the response of HB-ERD. Shorter block lengths lead to ringing effects, i.e. 'riding' oscillations on the ERD/ERS curve of twice the frequency of the investigated signal. This effect can be reduced if more epochs are taken into account. Longer blocks unnecessarily reduce the time resolution, which is expressed in a poorer agreement of the ERD with the actual envelope of the oscillatory activity. The added noise level of twice the variance of the signal did not change the behavior of any of the ERD/ERS variants significantly.

With respect to accuracy, it turns out that the ERD/ERS responses are not completely symmetrically distributed around the actual step latency. The ERD response reaches $50 \%$ of its maximal value about $10-30 \mathrm{~ms}$ later than the step has taken place. An important implication here, is that the $50 \%$ level of the ERD is quite a good estimate of the true onset of the actual oscillatory phenomenon, at least when this onset is brisk, as in our simulations. No statistically significant differences in accuracy between the various ERD/ERS methods could be found.

The step response, i.e. the time it takes for the ERD to climb from 5 to $95 \%$ of its maximal value, depends on the frequency of the signal, as one would expect. Again the type of ERD computation does not seem to play a major role. It is interesting to note that the step response is relatively slow, i.e. between 250 and $500 \mathrm{~ms}$. This means that in some cases it takes almost half a second for the ERD to reach $95 \%$ of its maximal value. An important implication of this finding is that short bursts of oscillatory activity (e.g. 50-100 ms) will produce smaller ERD effects than longer bursts (e.g. 200$500 \mathrm{~ms}$ ), which leave more time for the ERD measure to fully develop. Thus, effects that appear in the ERD as amplitude effects may in reality be caused by differences in length of the underlying oscillatory activity!

With respect to the sensitivity of the ERD measures, a striking and at first somewhat surprising finding is that sampling frequency plays a major role in the ability of the ERD to detect short-lasting trains of oscillatory bursts. The sensitivity is larger with higher sampling frequencies. For example, if we look at gamma activity $(40 \mathrm{~Hz})$ measured with an SNR of 1 in a total of 250 trials, bursts of $75 \mathrm{~ms}$ duration require a $10 \mathrm{kHz}$ sampling rate. Very short bursts $(<25 \mathrm{~ms})$ may even need $20 \mathrm{kHz}$. Only at these very high sampling frequencies, the HB-ERD seems to perform better than the block-ERD methods (Fig. 11). Bursts of 0.75 peri- 


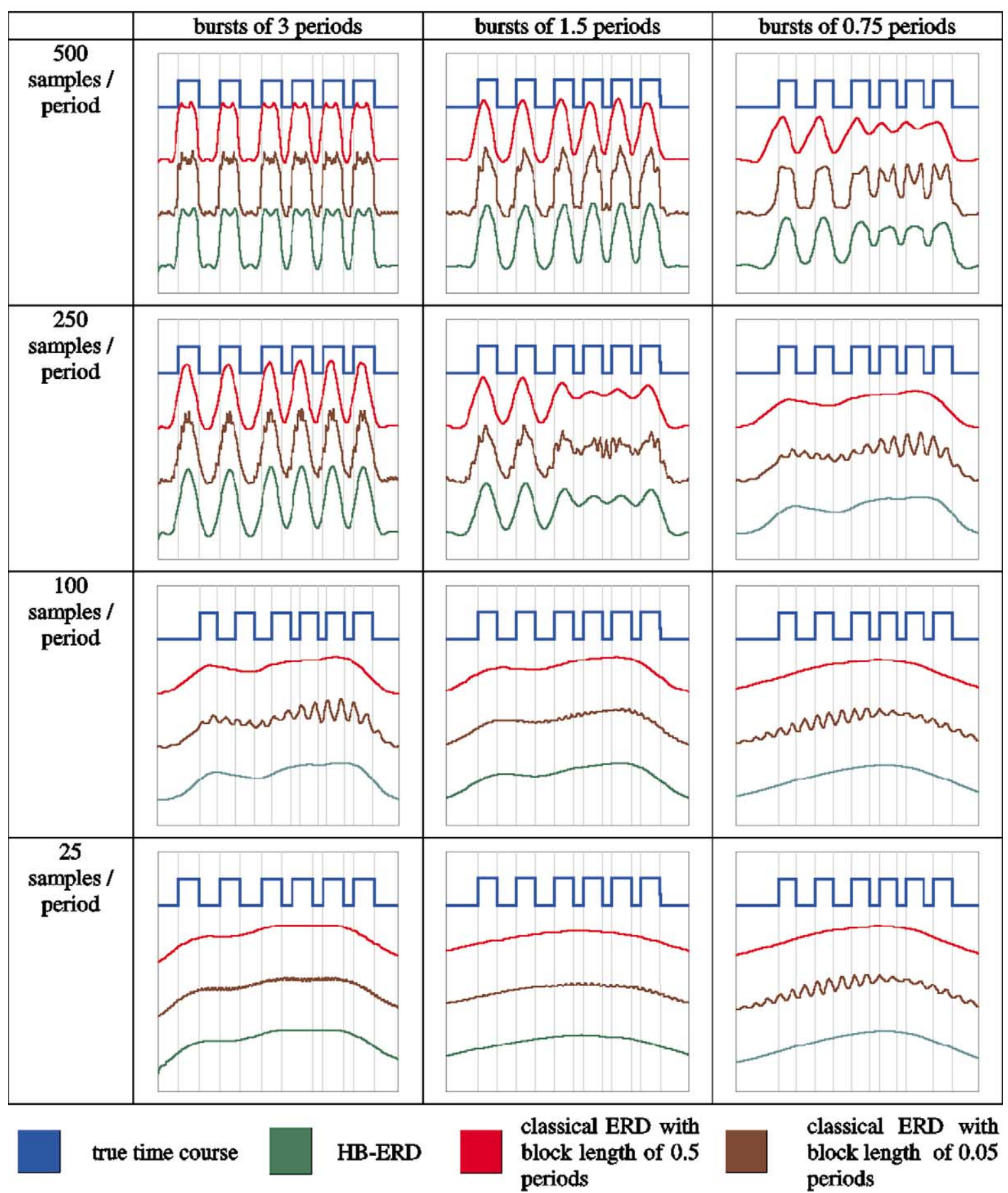

Fig. 11. ERD results for brief oscillatory bursts. The bursts contain 3, 1.5 , or 0.75 periods of the oscillation and are separated by 1 or 0.5 burst length. The signals are sampled with 500, 250, 100, or 25 samples per period of the oscillation. The oscillation amplitude during the bursts is twice the amplitude between them. The blue line indicates the true envelope of the burst signal.

ods of the signal (e.g. with a length of 18-20 ms for gamma frequencies) can be reliably resolved. When more moderate sampling rates are used, the different methods perform similarly. The dependence of the detectability of short bursts on the relative sampling rate (samples per period) can be attributed to the high frequencies that make up the sharp edges of the burst envelope. A too low sampling rate causes the ERD response not to follow the envelope closely enough and to smooth out the bursts.

Summarizing, one can state that the claim made by Clochon et al. (1996) that the HB-ERD has a millisecond time resolution can be definitely rejected on the basis of the present results. In terms of accuracy, HB-ERD is not superior to classical ERD with optimum block length $(0.5$ peri- 
Table 1

Summary of the results of the detection of brief oscillatory impulses in noisy data with HB-ERD

\begin{tabular}{|c|c|c|c|c|c|c|c|c|c|c|c|c|c|c|c|c|c|}
\hline & Samples per cycle & 500 & & & & 250 & & & & 100 & & & & 25 & & & \\
\hline & SNR & 10 & & 1 & & 10 & & 1 & & 10 & & 1 & & 10 & & 1 & \\
\hline & Trials & 250 & 25 & 250 & 25 & 250 & 25 & 250 & 25 & 250 & 25 & 250 & 25 & 250 & 25 & 250 & 25 \\
\hline Impulse [cycles] & Interval [cycles] & & & & & & & & & & & & & & & & \\
\hline 3 & 3 & $*$ & * & $*$ & * & * & $*$ & * & * & * & - & - & - & - & - & - & - \\
\hline 3 & 1.5 & $*$ & $*$ & $*$ & * & * & $*$ & $*$ & * & $*$ & - & $*$ & - & - & - & - & - \\
\hline 1.5 & 1.5 & $*$ & $*$ & $*$ & $*$ & $*$ & $*$ & $*$ & $*$ & - & - & - & - & - & - & - & - \\
\hline 1.5 & 0.75 & $*$ & $*$ & $*$ & $*$ & $*$ & $*$ & $*$ & $*$ & - & - & - & - & - & - & - & - \\
\hline 0.75 & 0.75 & $*$ & $*$ & $*$ & $*$ & - & - & - & - & - & - & - & - & - & - & - & - \\
\hline 0.75 & 0.375 & - & - & - & - & - & - & - & - & - & - & - & - & - & - & - & - \\
\hline
\end{tabular}

${ }^{\mathrm{a}}$ An asterisk indicates that all impulses of a certain kind could be significantly $(P<0.01$, Bonferoni-corrected $)$ distinguished from both the previous and the following inter-impulse interval.

ods), with one exception: if very high sampling frequencies are used, the HB-ERD might be somewhat better in resolving short-lasting oscillatory bursts than the block ERD methods. In all other cases, we can conclude that the HBERD performance is in the same range as that of classical ERD methods.

Basically, the Hilbert transform integrates the oscillation curve with a resolution of 0.5 periods (by connecting the peaks of the rectified curve), therefore the equivalence to classical ERD with a block length of 0.5 periods is plausible. For very short bursts, the integration blocks become longer than the bursts themselves and hence smooth them out.

Finally, despite the fact that HB-ERD has only minor advantages in terms of time resolution, it may be preferred over classical ERD methods. The most important reason for this is the fact that the method automatically adapts the time resolution to any frequency under investigation. This eliminates the need for manually adjusting block lengths to the frequency band under study, which is a time-consuming procedure that is rarely applied (cf. footnote 1 ). When classical block ERD is used, a block length of half the period of the slowest frequency component under study is recommended.

Finally, it should be noted that real MEG or EEG signals differ from the artificial signals we have used in our study. In particular, such signal contain broader frequency bands, which cannot be reduced to just one single frequency by even the sharpest filters. Moreover, the oscillation envelopes are normally not sharp jumps as in this study. Further investigations with more realistic signal properties might shed further light onto the performance of ERD techniques. The present study sought to reveal fundamental properties of the methods, especially with respect to the comparison between HB-ERD and classical block-based ERD.

\section{Acknowledgements}

The authors wish to thank Peter Hagoort, Jos van Berkum, and Burkhard Maess for their valuable comments on an earlier version of this paper.

\section{References}

Bastiaansen MCM, Böcker KBE, Cluitmans PJM, Brunia CHM. Eventrelated desynchronization related to the anticipation of a stimulus providing knowledge of results. Clin Neurophysiol 1999;110:250-260.

Clochon P, Fontbonne JM, Lebrun N, Etévenon PA. A new method for quantifying EEG event-related desynchronization: amplitude envelope analysis. Electroenceph clin Neurophysiol 1996;98:126-129.

Defebvre L, Bourriez J-L, Derambure P, Duhamel A, Guieu J-D, Destée A. Influence of chronic administration of L-DOPA on event-related desynchronization of mu rhythm preceding voluntary movement in Parkinson's disease. Electroenceph clin Neurophysiol 1998;109:161-167.

Etévenon P. Topological ERD and AM-EEG activation trajectories after hearing auditory stimulation. In: Witte H, Zwiener U, Schack B, Doering A, editors. Quantitative and topological EEG and MEG analysis, Jena-Erlangen: Druckhaus Mayer, 1997. pp. 355-357.

Klimesch W. EEG alpha and theta oscillations reflect cognitive and memory performance: a review and analysis. Brain Res Rev 1999;29: 169-195.

Klimesch W, Doppelmayr M, Wimmer H, Schwaiger J, Rohm D, Gruber W, Hutzler F. Theta band power changes in normal and dyslectic children. Clin Neurophysiol 2001;112:1174-1185.

Kolev V, Yordanova J, Schürmann M, Basar E. Event-related alpha oscillations in task processing. Clin Neurophysiol 1999;110:1784-1792.

Leocani L, Magnani G, Comi G. Event-related desynchronization during execution, imagination and witholding of movement. In: Pfurtscheller G, Lopes da Silva FH, editors. Event-related desynchronization, Handbook of Electroencephalography and Clinical Neurophysiology, revised series, vol. 6. Amsterdam: Elsevier, 1999. pp. 291-302.

Magnani G, Cursi M, Leocani L, Volonté MA, Locatelli T, Elia A, Comi G. Event-related desynchronization to contingent negative variation and self-paced movement paradigms in Parkinson's disease. Mov Disord 1998;13:653-660.

Neubauer AC, Freudenthaler HH, Pfurtscheller G. Intelligence and spatiotemporal patterns of ERD. Intelligence 1995;20:249-267.

Pfurtscheller G. Quantification of ERD and ERS in the time domain. In: Pfurtscheller G, Lopes da Silva FH, editors. Event-related desynchronization, Handbook of Electroencephalography and Clinical Neurophysiology, revised series, vol. 6. Amsterdam: Elsevier, 1999. pp. 89-106.

Pfurtscheller G, Aranibar A. Event-related cortical desynchronization detected by power measurements of scalp EEG. Electroenceph clin Neurophysiol 1977;42:817-826.

Pfurtscheller G, Berghold A. Patterns of cortical activation during planning of voluntary movement. Electroenceph clin Neurophysiol 1989;72: $250-258$.

Pfurtscheller G, Lopes da Silva FH. Event-related EEG/MEG synchronization and desynchronization: basic principles. Clin Neurophysiol 1999a; 110:1842-1857. 
Pfurtscheller G, Lopes da Silva FH, editors. Event-related desynchronization Handbook of Electroencephalography and Clinical Neurophysiology, revised series, vol. 6. Amsterdam: Elsevier, 1999b.

Pfurtscheller G, Neuper C. Event-related synchronization of mu rhythm in the EEG over the cortical hand area in man. Neurosci Lett 1994;174: 93-96.
Pfurtscheller G, Stancak A, Neuper C. Post-movement beta synchronization: a correlate of an idling motor area? Electroenceph clin Neurophysiol 1996;98:281-293.

Yordanova J, Kolev V. Event-related alpha oscillations are functionally related with P300 during information processing. NeuroReport 1998;9: 3159-3164. 